\title{
Plantas medicinais usadas pelos índios Tapebas do Ceará
}

\author{
Selene Maia de Morais*, Joana D’arc Pereira Dantas, Ana Raquel Araújo da Silva, \\ Everaldo Farias Magalhães
}

\author{
Curso de Química, Universidade Estadual do Ceará, Av. Paranjana 1700, Campus do Itaperi, \\ 60740-000, Fortaleza, CE, Brasil
}

\begin{abstract}
RESUMO: Este trabalho apresenta um levantamento das plantas medicinais utilizadas pelos índios Tapebas do Ceará, na tentativa de resgatar a cultura descaracterizada ao longo dos anos. Os parâmetros considerados foram a freqüência de uso das plantas e suas indicações populares para determinadas doenças, baseando-se em entrevistas realizadas nas comunidades localizadas no município de Caucaia, situado a $16 \mathrm{Km}$ de Fortaleza, capital do Ceará, em que foram mencionadas 63 plantas. Amostras das plantas foram coletadas e depositadas no Herbário Prisco Bezerra da Universidade Estadual do Ceará, onde foram identificadas. As plantas mais citadas foram agrupadas de acordo com seus dados químico, farmacológico e/ou toxicológico encontrados na literatura científica. A pesquisa mostra que a comunidade indígena dos Tapebas faz uso de muitas espécies vegetais sem dados químico e farmacológico registrados, bem como de outras que já foram alvo de pesquisa científica, mas que necessitam ainda de estudos complementares para garantir segurança para um uso geral e preparação de fitoterápicos.
\end{abstract}

Unitermos: Plantas medicinais, índios, Tapebas, Ceará.

\begin{abstract}
Ethno-medicinal plants of Tapeba Indians from the State of Ceará - Brazil”. This work presents a survey of the medicinal plants used by Tapebas Indians from the state of Ceará - Brazil, trying to rescue their culture which is vanishing through the years. Several interviews were performed at the indian's community located at municipality of Caucaia, $16 \mathrm{Km}$ away from Fortaleza, Capital city of the state of Ceará. A total of 63 plants were cited. The interviews were based on the frequency that the plants are used and their respective uses. Samples of the plants were collected and classified by botanists of the Prisco Bezerra Herbarium of the Universidade Federal do Ceará. A literature survey was carried out with all known plants to obtain chemical, pharmacological and toxicological informations. The results show that the Tapebas use plants with no reported chemical and pharmacological informations and other plants which were already studied but need yet more studies to assure complete safety for a general use and production of phytoterapics.
\end{abstract}

Keywords: Medicinal plants, indians, Tapebas, Ceará.

\section{INTRODUÇÃO}

Os índios Tapebas formam um grupo étnico que habita o município de Caucaia, a $16 \mathrm{Km}$ de Fortaleza. Eles compõem 17 comunidades organizadas na "Associação das Comunidades do Rio Ceará”. Cerca de 200 famílias já estão com suas terras delimitadas e foi feito o levantamento de pessoas residentes que não são indígenas e fizeram benfeitorias na área, através da FUNAI. Eles aguardam a conclusão do processo demarcatório de suas terras e de sua identificação oficial, enquanto continuam a habitar áreas diferentes, as quais constituem aglomerados populacionais de densidade e composição populacional bem como disposição espacial bastante contrastantes (Cordeiro, 1989). Este autor relata ainda que os índios já não falam sua língua nativa, esqueceram muito dos costumes cultivados pelos ancestrais e suas atividades econômicas são o pescado no rio Ceará e no manguezal, o comércio de artesanatos e ainda atividades sazonais e mal remuneradas. A falta de recursos os faz recorrer à utilização de plantas medicinais.
O presente trabalho teve como objetivo identificar as plantas mais utilizadas pelos índios Tapebas do Ceará, das quais muitas tiveram suas ações terapêuticas validadas, tendo seu uso assegurado por estudos científicos realizados, estando incluídas no Projeto Farmácias Vivas.

\section{MATERIAL E MÉTODOS}

O trabalho foi realizado no município de Caucaia a $16 \mathrm{Km}$ de Fortaleza, envolvendo entrevistas com 45 moradores da comunidade indígena, inclusive com o Pajé. O modelo de questionário utilizado encontra-se no Anexo 1. Para o desenvolvimento deste trabalho foram realizadas as seguintes etapas:

a) obtenção dos dados socio-econômicos sobre os índios Tapebas no Instituto Histórico do Ceará, Biblioteca Estadual do Ceará e na comunidade indígena, através de um projeto organizado pelos próprios índios, chamado "Escola diferenciada Tapeba";

b) levantamento das plantas utilizadas como medicinais 
na comunidade indígena através de entrevistas e preenchimento de formulários adequados;

c) identificação das espécies coletadas, que foram depositadas no Herbário Prisco Bezerra do Departamento de Biologia da Universidade Federal do Ceará;

d) revisão na literatura científica para cada espécie, obtendo informações sobre o uso popular, a composição química, a atividade farmacológica estabelecida e a toxicidade, através de levantamentos em periódicos científicos e livros específicos desta área (Matos et al., 2001; Matos, 2000; Braga, 1976).

\section{RESULTADOS E DISCUSSÃO}

As plantas mais referidas pelos índios Tapebas resultaram, após a identificação, em 57 táxons identificados até espécie, dois identificados apenas pelo gênero e quatro plantas não identificadas, perfazendose, então, um total de 63 táxons. As famílias com maior número de espécies mencionadas foram Lamiaceae (5), Liliaceae (5), Anacardiaceae (4), Euphorbiaceae (4) e Fabaceae sensu lato (5).

As informações sobre as plantas mencionadas pelos índios foram agrupadas na forma de tabelas. Na tabela 1 , as plantas estão listadas segundo o nome popular, nome científico e família botânica correspondentes. Na tabela 2 as plantas são apresentadas pelo seu nome popular, além de serem relacionadas quanto a sua indicação popular, parte utilizada, posologia e modo de uso. Das 63 plantas referidas somente 49 tiveram suas ações relatadas, pois foram mais citadas. Tais informações foram descritas de acordo com os relatos dos índios entrevistados, utilizando um formulário de entrevista.

As espécies mais utilizadas pelos índios foram agrupadas em três categorias distintas. A categoria 1 agrupa nove plantas cujo uso é considerado validado. A categoria 2 contém cinco plantas cujas atividades farmacológicas foram bem determinadas. No entanto, estas apresentam limitações no uso devido à toxicidade quando usadas em altas doses. A categoria 3 agrupa nove plantas que são pouco conhecidas quimicamente, mas que contam com alguma atividade biológica detectada. A seguir estão listadas as categorias estabelecidas e as informações obtidas para as espécies na pesquisa bibliográfica, a qual enfocou a constituição química e a atividade biológica.

\section{Categoria 1. Plantas de uso validado}

Amburana cearensis (Allemão) A. C. Sm. (Cumarú) - Seu principal componente químico é a cumarina, que é responsável, juntamente com outras substâncias, pela atividade broncodilatadora determinada experimentalmente, validando cientificamente o uso popular e o tratamento caseiro, especialmente em crianças e idosos. Glicosídeos fenólicos encontrados em sua composição apresentaram atividade antimalárica, antiprotozoária, antifúngica e antibacteriana in vitro (Bravo et al., 1999).

Astronium urundeuva (Allemão) Engl. (Aroeira-dosertão) - A entrecasca da aroeira é largamente usada na medicina popular do nordeste brasileiro para o tratamento de problemas dermatológicos e ginecológicos (Matos, 2000), sendo verificadas experimentalmente sua atividade cicatrizante (Viana et al., 1995), antiinflamatória e antiulcerogênica (Menezes; Rao, 1986). Estudos com os extratos dos brotos e renovos do caule de $A$. urundeuva permitiu comprovar a existência de chalconas diméricas antiinflamatórias (Viana et al., 2003; Bandeira et al., 1994) e taninos com ação analgésica e antiinflamatória (Viana et al., 1997). Foram isolados ainda compostos mais apolares como cicloeucalenol e cicloeucalenona a partir do extrato hexânico da entrecasca, que apresentam atividade antioxidante (Dantas, 2003).

Cymbopogon citratus (DC.) Stapf (Capim-santo) - A ação calmante e espasmolítica leve comprovada é atribuída ao citral presente no óleo essencial (Matos, 2000), onde também é encontrado o mirceno, responsável pela sua ação analgésica (Lorenzetti et al., 1991).

Hybanthus ipecacuanha (L.) Baill. (Ipecacuanha) - A atividade antinociceptiva, antiinflamatória e broncodilatadora desta espécie foi observada em estudos preliminares, sendo tais ações atribuídas à presença de cumarina, um dos princípios ativos em sua composição dentre outros compostos (por exemplo, taninos e flavonóides), justificando seu uso popular tradicional no tratamento de doenças do aparelho respiratório (Leal et al., 2000).

Hyptis suaveolens (L.) Poit. (Bamburral) - Seu óleo essencial apresenta uma variação na composição química, de acordo com a localização geográfica. Os sesquiterpenos existentes nessa planta são produzidos em maior quantidade em baixas altitudes e latitudes (Azevedo et al., 2002), sendo ainda encontrados constituintes triterpênicos nas folhas (Raja Rao et al., 1990). A presença de alto teor de cineol no óleo essencial das folhas permite o uso como antigripal na forma de inalação com vapor d'água.

Kalanchoe brasiliensis Cambess. (Courama) - Tem largo uso popular no tratamento de furúnculos. Por via oral, o sumo é usado puro nos casos de inflamações ovarianas e uterinas ou misturado com malvarisco ou outras plantas na preparação de xaropes para tosse. Estudos químicos determinaram a presença de vários esteróis (Akihisa et al., 1991), sendo comprovada sua atividade imunomoduladora, antiinflamatória (Ibrahim et al., 2002), além de uma ação leishimanicida potencialmente útil contra Leishmaniose cutânea (Bergman et al., 1992).

Ocimum gratissimum L. (Alfavaca) - As ações biológicas são justificadas pelo seu óleo essencial contendo eugenol, que confere à planta um poder anti-séptico, e ainda o 1,8cineol, um princípio balsâmico responsável pelo emprego eficaz da planta na preparação de banhos anti-gripais em crianças (Matos, 2000). Estão presentes também na 
composição do óleo, metil-eugenol, $\gamma$-selineno, chavicol, nerol, timol, trans-cariofileno, terpineno, canfeno, carvacrol, $\alpha$ e $\beta$-pineno, entre outros componentes minoritários (Matos et al., 2001). Alguns dos usos populares foram verificados cientificamente, como a atividade relaxante sobre o músculo liso do intestino (Madeira et al., 2002) e a ação fungicida contra quatro espécies do gênero Candida (Nakamura et al., 2004).

Spondias mombim Jacq. (Cajazeira) - Verificou-se sua atividade contra o vírus Coxsakii B e Herpes Simplex I, responsáveis pelas crises de aftas dolorosas e pela herpes labial, respectivamente, atribuída à presença de quatro substâncias, sendo duas do grupo dos elagitaninos, geraniina e galoilgeraniina, cuja atividade antivirótica ocorre em uma diluição específica (Corthout et al., 1991) e dois ésteres do ácido caféico ativos (Corthout et al., 1992).

Tabebuia serratifolia (Vahl) G. Nicholson (Pau-d'arcobranco) - O seu princípio ativo é o lapachol, presente tanto nas cascas do caule como na serragem da madeira (Matos, 2000), conferindo à planta uma atividade antiinflamatória verificada experimentalmente (Almeida et al., 1990). Várias espécies de Tabebuia contêm na casca e no cerne do caule quantidades variáveis dessa e outras substâncias similares, dotadas de forte atividade antimicrobiana, antialérgica, cicatrizante e antitumoral (Oliveira et al., 1990).

\section{Categoria 2. Plantas de toxicidade estabelecida}

Acanthospermum hispidum DC. (Delegado) - As folhas desta espécie são usadas na medicina popular no tratamento de doenças infecciosas. No entanto, a base molecular e biológica desta atividade é ainda pouco conhecida. Summerfield et al. (1997) investigaram e caracterizaram uma possível atividade antiviral desta espécie, especialmente contra determinados vírus da herpes. A atividade antimicrobiana das folhas também foi investigada, revelando eficácia contra diversos tipos de bactérias patogênicas (Fleischer et al., 2003). Estudos em animais evidenciaram ação abortiva e teratogênica (Lemonica; Alvarenga, 1994). Hussain et al. (1990) verificaram a presença de terpenóides e polifenóis.

Aloe vera L. (Babosa) - A babosa tem ação cicatrizante, antibacteriana, antifúngica e antivirótica pela presença das antraquinonas como aloenina, barbaloína e isobarbaloína em sua composição química (Kuzuya et al., 2001; Steinert et al., 1996). Tais propriedades justificam seu uso popular, mas por causa da sua ação nefrotóxica em doses altas, não deve ser usada como lambedor, pois nessa preparação o teor de seu princípio predominante é aumentado e pode causar severa crise de nefrite aguda (Matos, 2000). Tendo grande capacidade de regenerar tecidos lesados, o gel que a planta contém, onde estão presentes alguns tipos de glicoproteínas e polissacarídeos, pode ser usado seguramente sobre a pele na forma de emplastro (Reynolds; Dweck, 1999).
Cajanus cajan (L.) Millsp. (Feijão-cuandu) - Estudos em animais evidenciaram ação abortiva e teratogênica ( Lemonica; Alvarenga,1994).

Chenopodium ambrosioides var. anthelminticum (L.) A. Gray (Mastruço ou mastruz) - O uso do óleo essencial desta espécie como anti-helmíntico para humanos foi substituído por medicamentos mais modernos e seguros devido a sua toxicidade (Paget, 1926). O óleo de quenopódio, como é chamado, contem ascaridol, um potente anti-helmíntico que tem sido também responsável por fatalidades em humanos (Macdonald et al., 2004). De acordo com Salant e Mitchell (1916), quando injetado na corrente sanguínea de animais (coelhos, gatos e cachorros), o óleo provoca uma depressão da circulação, respiração e movimentos intestinais. Tal espécie continua a ser usada no tratamento de vermes do intestino e outras doenças em humanos, com aparente sucesso (Quinlan et al., 2002), não sendo encontrado na literatura científica relato de casos de severos envenenamentos ou mortes a partir da infusão aquosa de C. ambrosioides (Kliks, 1985). No Nordeste do Brasil onde é largamente utilizado, as folhas do mastruz sâo batidas no liquidificador com leite para tratamentos de gripe e, para ajudar na recuperação de fraturas ósseas de animais, as folhas são amarradas no local fraturado.

Phyllanthus amarus Schum. et Thonn. (Quebra-pedra) - Várias atividades biológicas têm sido relatadas para esta planta como inibitória da replicação do vírus HIV (Notka et al., 2004), anti-alodínica e anti-endematogênica do extrato e lignanas (Kassuya et al., 2003), inibição de lesão gástrica e inflamação (Raphael; Kuttan, 2003), antitumoral e anticarcinogênica (Rajeshkumar et al., 2002) Análise química tem evidenciado a presença de vários constuituintes pertencentes às classes dos taninos hidrolisáveis (Foo; Wong, 1992), lignanas e alcalóides pirrolizidínicos, mas ainda não se sabe qual é seu principio ativo. É considerado tóxico em altas doses devido a presença dos alcalóides pirrolizidínicos (Matos, 2000).

\section{Categoria 3. Plantas pouco conhecidas quimicamente} e com alguma atividade biológica comprovada

Bauhinia ungulata L. - (Mororó ou pata de vaca) - Embora sua atividade hipoglicemiante tenha sido comprovada em vários experimentos (Morais; Andrade, 1980), um único estudo químico registra a presença de insulina nos cloroplastos das células foliares (Lorenzi; Matos, 2002).

Bixa orellana L. (Urucum) - Desde os tempos mais remotos os indígenas brasileiros usam o pigmento do urucum como proteção contra insetos e queimaduras por exposição ao sol. Também amplamente usado como corante dos alimentos na cozinha nordestina. As sementes são referidas na literatura etnofarmacológica como medicação estomáquica, tonificante do aparelho gastrintestinal, andiarréica, antifebril bem como para o tratamento caseiro das palpitações do coração, crises

Rev. Bras. Farmacogn. Braz J. Pharmacogn. 15(2):abr/jun. 2005 
de asma, coqueluche e gripe. Empregado na medicina popular, na forma de chá ou maceradas em água fria ou como xarope nos casos de faringitee bronquite. Na semente ocorre um óleo rico em all-E-geranilgeraniol, monoterpenos e sesquiterpenos oxigenados, além dos carotenóides bixina e norbixina responsáveis pela sua cor e alfa e beta-caroteno (Lorenzi; Matos, 2002).

Boerhavia hirsuta Wiild. (Pega-pinto) - As folhas por infusão são usadas para doenças do fígado, do baço, dos rins, inflamação, febre (Coelho; da Silva). Extratos hexânico e etanólico desta planta revelaram uma fraca atividade antimalárica ( Krettli et al., 2001).

Cecropia pachystachia Trec. (Torém) - Suas ações diurética e anti-hipertensiva foram demonstradas experimentalmente, mas seus princípios ativos não são conhecidos. Outra espécie C. glaziovii Sneth., encontrada no Centro-sul do Brasil, apresenta a iso-vitexina, componente isolado da fração do extrato dotado de ação anti-hipertensiva (Matos, 2000).

Hibiscus esculentus L. (Quiabo) - O extrato aquosoetanólico do quiabo apresenta atividade antioxidante e os constituintes identificados para esta ação foram 3-O-xilosil-quercetina, 3-O-glicosil-quercetina e (-)epigalocatequina (Shui; Peng, 2004). Foi registrado também o efeito anti-ulcerogênico do extrato aquoso (Gürbüz et al., 2003)

Justicia pectoralis Jacq. (Anador) - Esta é uma dentre várias espécies pertencentes a diferentes famílias botânicas do nordeste brasileiro que têm em comum a presença de cumarina em sua composição. Tais espécies são referidas na medicina popular no tratamento de doenças do aparelho respiratório (Rocha, 1945; Braga, 1976; Correa, 1984). Um estudo fitoquímico das folhas mostrou, além da presença de cumarinas, flavonóides, saponinas e taninos, sendo sua atividade antiinflamatória e broncodilatadora demonstrada experimentalmente (Leal et al., 2000).

Plumbago scandens L. (Louco) - A planta apresenta a naftoquinona plumbagina que demonstrou atividade anticâncer, leishmanicida e bactericida, sendo esta substância também efetiva contra insetos (Paiva et al., 2004)

Sansevieria cylindrica Bojer (Rabo-de-tatu) - É relatada a presença de uma saponina esteroidal sem efeitos hemolíticos in vitro que demonstrou inibição na permeabilidade capilar (Antunes et al., 2003).

Ximenia americana L. (Ameixa) - Tanto o extrato da casca, bem como das folhas e dos talos tiveram sua atividade fungicida comprovada experimentalmente (Omer; Elnima, 2003), porém pouco se sabe sobre sua composição química.

Os índios Tapebas, assim como a população em geral faz uso de plantas medicinais no tratamento de suas doenças. No entanto, para muitas das espécies utilizadas não existem trabalhos científicos que garantam seu uso seguro, por meio do conhecimento químico, farmacológico e da toxicidade destas plantas. Outro fato observado foi o da não concordância do conhecimento científico atual com o uso popular, como é o caso da boa-noite-branca, utilizada pelos índios Tapebas contra dor, enquanto que as pesquisas indicam a ação de alguns de seus alcalóides contra determinados tipos de câncer. Algumas das plantas referidas neste levantamento etnobotânico, após estudos, já foram incluídas no projeto "Farmácias Vivas”, pelo Dr. Francisco José de Abreu Matos como o cumaru, aroeira do sertão, capim santo, ipecacuanha, bamburral, courama, alfavaca, cajazeira e pau-d’arco-branco.

\section{AGRADECIMENTOS}

Os autores agradecem a Fundação de Apoio ao Desenvolvimento Científico e Tecnológico do Estado do Ceará (FUNCAP) pelas bolsas de Iniciação Científica.

\section{REFERÊNCIAS}

Akihisa T, Kokke WCMC, Tamura T 1991. Sterols of Kalanchoe pinnata: first report of the isolation of both epimers of 24-alkyl-d-25-sterols from higher plants. Lipids 26: 660-665.

Almeida ER, Filho AAS, Santos ER, Lopes CAC 1990. Antiinflammatory action of lapachol. $J$ Ethnopharmacol 29: 239-241.

Antunes SA, Da Silva BP, Parente JP, Valente AP 2003. A new bioactive steroidal saponin from Sansevieria cylindrica. Phytother Res 17: 179-182.

Azevedo NR, Campos IFP, Ferreira HD, Pontes TA, Seraphin IC, Realino De Paula I, Santos SC, Ferri PH 2002. Essential oil chemotypes in Hyptis suaveolens from Brazilian Cerrado. Biochem Syst Ecol 30: 205-216.

Bandeira MAM, Matos FJA, Braz-Filho R 1994. New chalconoid dimers from Myracrodruon urundeuva. Nat Prod Lett 4: 113-120.

Bergman R, Moraes VGL, Silva S 1992. Ação do extrato aquoso de Kalanchoe pinnata (Saião) sobre o parasita Leishmania - I, estudo in vivo. XII Simpósio de Plantas Medicinais do Brasil, Curitiba, PR.

Braga, R 1976. Plantas do Nordeste especialmente do Ceará, Imprensa Oficial, 3a edição, Fortaleza.

Bravo JA, Sauvain M, Gimenez A, Munoz VO, Callapa J, Le Men-Olivier L, Massiot G, Lavand C 1999. Bioactive phenolic glycosides from Amburana cearensis. Phytochemistry 50: 71-74.

Coelho MFB, Da Silva AC. Plantas de uso medicinal nos municípios de Pontes e Lacerda e de Comodoro, Mato Grosso, Brasil. http://www.ufmt.br/agtrop/revista7/ doc/05.htm acessada em novembro de 2004.

Cordeiro J 1989. Os Índios no Siará: Massacre e resistência, Fortaleza: Hoje- Assessoria em Educação.

Correa PM 1984. Dicionário de plantas úteis do Brasil e das exóticas cultivadas. Ministério da Agricultura, Instituto Brasileiro de Desenvolvimento, Florestal, Brasil 5: 474-475.

Corthout J, Pieters L, Claeys M, Vanden Berghe D, Vlietinck A 1992. Antiviral caffeoyl esters from Spondias mombin. Phytochemistry 31: 1979-1981.

Corthout J, Pieters L, Claeys M, Vanden Berghe D, Vlietinck A 1991. Antiviral ellagitannins from Spondias mombin. Phytochemistry 30: 1129-1130. 
Dantas JDP 2003. Contribuição científica à medicina tradicional dos Tapebas do Ceará: Astronium urundeuva (Allemão) Engl. - (aroeira-do-sertão), Fortaleza, Monografia de graduação do curso de química da Universidade Estadual do Ceará.

Fleischer TC, Ameade EPK, Sawer IK 2003. Antimicrobial activity of the leaves and flowering tops of Acanthospermum hispidum. Fitoterapia 74: 130132.

Foo LY, Wong HY 1992. Phyllanhtusiin D, an unusual hydrolysable tannin from Phyllanthus amarus. Phytochemistry 31: 711-713.

Gürbüz I, Üstün O, Yesilada E, Sezik E, Kutsal O 2003. Antiulcerogenic activity of some plants used as folk remedy in Turkey. J Ethnopharmacol 88: 93-97.

Hussain RA, Lin Y, Poveta LJ, Bordas E, Chung BS, Pezzuto JM, Soejabto DD, Kinghom AD 1990. Plant-derived sweetening agents: Saccharide and polyol constituents of some sweet-tasting plants. J Ethnopharmacol 28: 103-115.

Ibrahim T, Cunha JMT, Madi K, Fonseca LMB, Costa SS, Koatz VLG 2002. Immunomodulatory and antiinflammatory effects of Kalanchoe brasiliensis. Int Immunopharmacol 875-883.

Kassuya CAL, Silvestre AA, Rehder VLG, Calixto JB 2003. Anti-allodynic and anti-oedematogenic properties of the extract and lignans from Phyllanthus amarus in models of persistent inflammatory and neurophatic pain. Eur J Pharmacol 478: 145-153.

Kliks MM 1985. Studies on the traditional herbal anthelmintic Chenopodiumambrosioides L.: ethnopharmacological evalution and clinical fields trials. Social Science and Medicine 21: 879-886.

Krettli AU, Andrade-Neto VF, Brandão ML, Ferrari WMS 2001. The search for new antimalarial drugs from plants used to treat fever and malaria or plants randomly selected: a review. Mem Inst Oswaldo Cruz 96: 10331042.

Kuzuya H, Tamai I, Beppu H, Shimpo K, Chihara T 2001. Determination of aloenin, barbaloin and isobarbaloin in Aloe species by micellar electrokinetic chromatography. J Cromatogr 752: 91-97.

Leal LKAM, Ferreira AAG, Bezerra GA, Matos FJA, Viana GSB 2000. Antinoceptive, antiinflamatory and broncodilator activities of Brazilian medicinal plants containing coumarin: a comparative study. $J$ Ethnopharmacol 70: 151-159.

Lemonica LP, Alvarenga CMD 1994. Abortive and teratogenic effect of Acanthospermum hispidum DC. and Cajanus cajan (L.) Millps in pregnants rats. J Ethnopharmacol 43: 39-44.

Lorenzetti BB, Souza GEP, Sarti SJ, Santos Filho D, Ferreira SH 1991. Myrcene mimics the peripheral analgesic activity of lemongrass tea. $J$ Ethnopharmacol 34: 4348.

Lorenzi H, Matos FJA 2002. Plantas medicinais do Brasil : nativas e exótica cultivadas, Nova Odessa, SP : Instituto Plantarum.

Macdonald D, Vancrey K, Harrison P, Rangachari PK, Rosenfeld J, Warren C, Sorger G 2004. Ascaridoleless infusions of Chenopodium ambrosioides contain a nematocide(s) that is(are) not toxic to mammalian smooth muscle. J Ethnopharmacol 92: 215-221.
Madeira SVF, Matos FJA, Cardoso JHL, Criddle DN 2002. Relaxant effects of the essential oil of Ocimum gratissimum Linn. on isolated ileum of the guinea pig. J Ethnopharmacol 81: 1-4.

Matos FJA 2000. Plantas medicinais - Guia de seleção e emprego de plantas usadas em fitoterapia no Nordeste do Brasil, 2 edição: Imprensa universitária da UFC, Fortaleza.

Matos FJA, Viana GSB, Bandeira MAM 2001. Guia fitoterápico, $2^{a}$ edição, Fortaleza.

Menezes AMS, Rao VS 1986. Anti-ulcerogenic activity of Astronium urundeuva. Fitoterapia 57: 55-57.

Morais KBM, Andrade MNF 1980. Acompanhamento do uso da tintura das folhas de mororó, a 20\% (Bauhinia ungulata L.) em diabéticos. XVI Simpósio de Plantas Mediciais do Brasil, Florianópolis.

Nakamura CV, Ishida K, Faccin LC, Filho BPD, Cortez DAG, Rozental S, Souza W, Nakamura TU 2004. In vitro activity of essential oil from Ocimum gratissimum L. against four Candida species. Res Microbiol 155: 579-586.

Notka F, Meier G, Wagner R 2004. Concerted inhibitory activities of Phyllanthus amarus on HIV replication in vitro and ex vivo. Antivir Res 64: 93-102.

Oliveira AB, Raslan DS, Miraglia MCM, Mesquita AAL, Zani CL, Ferreira DT, Maia JGS 1990. Estrutura química e atividade biológica de naftoquinonas de Bignoniaceas brasileiras. Quím Nova 13: 302-307.

Omer MEFA, Elnima EI 2003. Antimicrobial activity of Ximenia americana. Fitoterapia 74: 122-126.

Paget H 1926. The determination of ascaridole in Chenopodium oil. Analyst 51: 170-176.

Paiva SR, Lima LA, Figueiredo MR, Kaplan AC 2004. Plumbagin quantification in roots of Plumbago scadens L. obtained by different extraction techniques. An Acad Bras Ciênc, 76: 499-504.

Quinlan MB, Quinlan RJ, Nolan JM 2002. Ethnophysiology and herbal treatment of intestinal worms in Dominica, West Indies. J Ethnopharmacol 80: 75-83.

Raja Rao KV, Rao LJM, Prakasa Rao NS 1990. An a-ring contracted triterpenoid from Hyptis suaveolens. Phytochemistry 29: 1326-1329.

Rajeshkumar NV, Joy KL, Kuttan R, Ramsewak RS, Nair MG, Kuttam R 2002. Antitumour and anticarcinogenic activity of Phyllanthus amarus extract. $J$ Ethnopharmacol 81: 17-22.

Raphael KR, Kuttan R 2003. Inhibition of experimental gastric lesion and inflammation by Phyllanthus amarus extract. J Ethnopharmacol 87: 193-197.

Reynolds T, Dweck AC 1999. Aloe vera leaf gel: a review update. J Ethnopharmacol 68: 3-37.

Rocha D 1945. Formulário terapêutico de plantas medicinais cearenses, nativas e cultivadas. Progresso, Fortaleza.

Salant WF, Mitchell CW 1916. Influence of oil of Chenopodium on intestinal contractility. Am J Phys 39: 37-52.

Shui G, Peng LL 2004. An improved method for the analysis of major antioxidants of Hibiscus esculentus L. $J$ Chromatogr A 1048: 17-24.

Steinert J, Khalaj S, Rimpler M 1996. High-performance liquid chromatographic separation of some naturally occurring naphthoquinones and anthraquinones. $J$ Chromatogr A 723: 206-209. 
Summerfield A, Keil GM, Mettenleiter TC, Rziha HJ, Saalmüller A 1997. Antiviral activity of an extract from leaves of the tropical plant Acanthospermum hispidum. Antiv Res 36: 55-62.

Viana GSB, Bandeira MAM, Matos FJA 2003. Analgesic and antiinflammatory effects of chalcones isolated from Myracrodruon urundeuva Allemão. Phytomedicine 10: 189-195.

Viana GSB, Bandeira MAM, Moura LC, Souza-Filho MVP, Matos FJA, Ribeiro RA 1997. Analgesic and antiinflammatory effects of the tannin fraction from Myracrodruon urundeuva Fr. All. Phytother Res 11: 118-122.

Viana GSB, Matos FJA, Bandeira MAM, Rao VS 1995. Aroeira-do-sertão (Myracrodruon urundeuva Fr. All.): estudo botânico, farmacognóstico, químico e farmacológico, $2^{\mathrm{a}}$ edição revisada e ampliada, Fortaleza, Edições UFC. 
Anexo 1. Formulário de entrevistas

FORMULÁRIO DE ENTREVISTA

PLANTAS MEDICINAIS DE USO DOS ÍNDIOS TAPEBAS

LOCAL DA COLETA DA INFORMAÇÃO

MUNICÍPIO

UF

LOCALIDADE

DADOS DO INFORMANTE

NOME

HOMEM ( ) MULHER ( ) IDADE

DADOS DA PLANTA E DE SEU USO

PLANTA (outros dados do verso).

PARTE USADA VIA

PREPARAÇ̃̃O

INDICAÇÃO (vide o verso)

\section{DADOS COMPLEMENTARES}

QUEM USA? (Homem, mulher ou criança)

MODO DE USAR

FREQUÊNCIA DA DOSE:

RESULTADOS

\section{ENTREVISTADOR(A)}

NOME COMPLETO

DATA ASSINATURA

MATERIAL BOTÂNICO

COLETOR Coleta \#

DATA E LOCAL DE COLETA

DATA MUNICÍPIO, ESTADO

\section{IDENTIFICAÇÃO BOTÂNICA}

NOME VULGAR a) NO LOCAL DA COLETA

b) NO LOCAL DA INFORMAÇÃO ESPÉCIE FAMÍLIA

DETERMINADOR: DATA

\section{DADOS COMPLEMENTARES}

HÁBITO DE CRESCIMENTO SUBSTRATO COR DA FLOR: VEGETAÇÃO ASSOCIADA COR DO FRUTO 
Tabela 1. Nomes populares, científicos e família de todas as plantas referidas nas entrevistas, realizadas na comunidade indígena dos índios Tapebas do Ceará

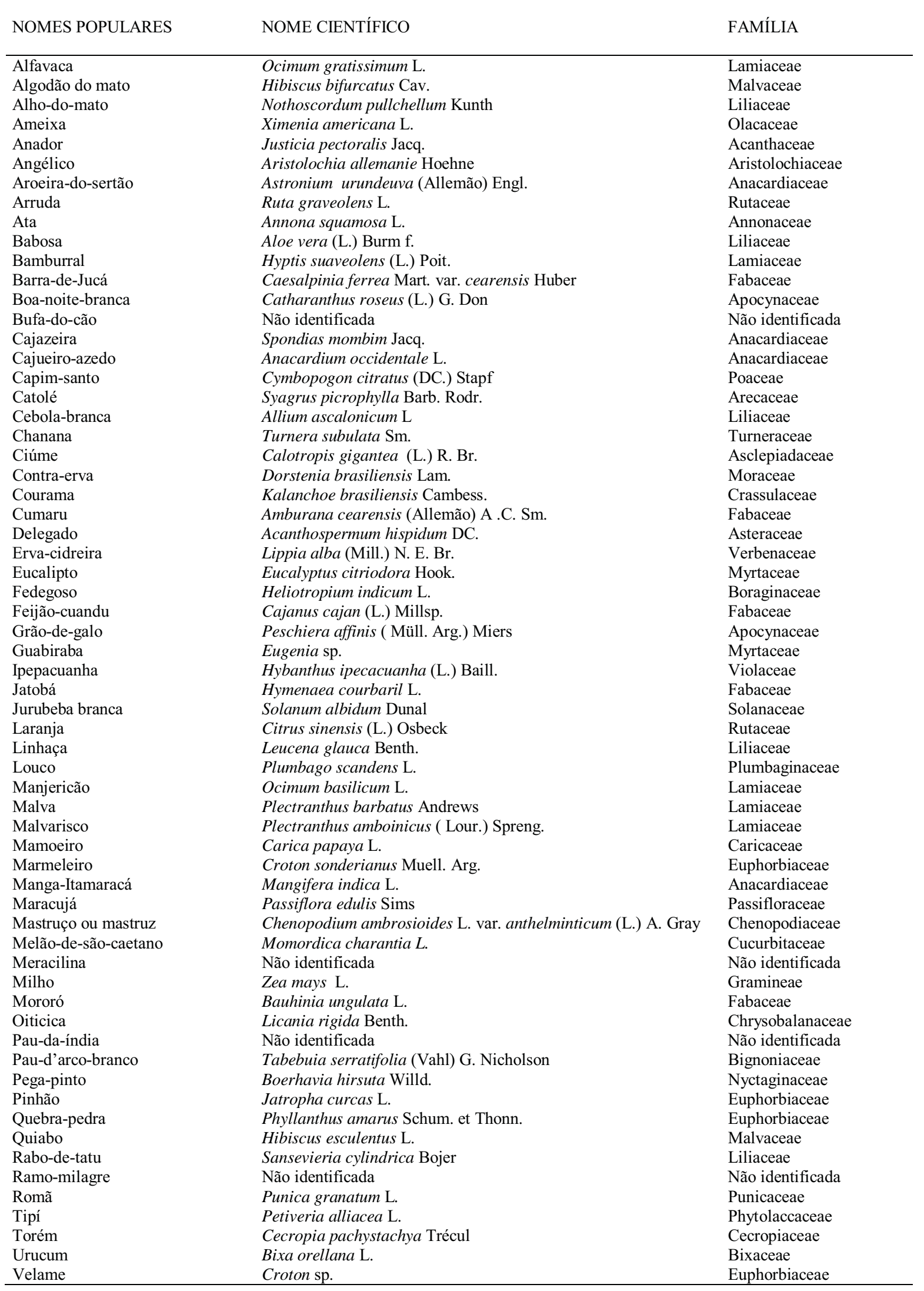


Tabela 2. Relação das plantas referidas pelos índios Tapebas do Ceará de acordo com o emprego que fazem, algumas vezes se valendo da associação conjunta de plantas

\begin{tabular}{|c|c|c|c|c|}
\hline NOME DA PLANTA & $\begin{array}{l}\text { PARTE } \\
\text { UTILIZADA }\end{array}$ & MODO DE USO & POSOLOGIA & INDICAÇÃO POPULAR \\
\hline Alfavaca e eucalipto & Folhas & Chá & 3 à 4 litros & Assepsia \\
\hline Algodão-do-mato & Algodão & Queimado & $\begin{array}{l}\text { Inalar a fumaça ao } \\
\text { sintoma de dor }\end{array}$ & Dor de cabeça \\
\hline $\begin{array}{l}\text { Alho-do-mato e cebola- } \\
\text { branca }\end{array}$ & Raiz & Chá & 3 vezes ao dia & Asma \\
\hline Anador & Folhas & Chá & 3 vezes ao dia & Hipertensão \\
\hline $\begin{array}{l}\text { Aroeira-do-sertão e } \\
\text { ameixa }\end{array}$ & Casca do caule & $\begin{array}{l}\text { Extrato hidroalcoól. } \\
\text { (com cachaça) }\end{array}$ & Até passar os sintomas & Feridas \\
\hline Arruda & Folhas & Chá & 3 vezes ao dia & Dores \\
\hline Ata & Casca do fruto & $\begin{array}{l}\text { Faz-se um emplastro } \\
\text { com o pó }\end{array}$ & Até passar os sintomas & Estancar o sangue \\
\hline Bamburral & Folhas & Chá & Tomar 3 vezes ao dia & Má digestão \\
\hline Barra-de-jucá & Semente & $\begin{array}{l}\text { Extrato hidroalcoól. } \\
\text { (com cachaça) }\end{array}$ & Até passar os sintomas & Sangramentos externos cãibra \\
\hline Boldo & Folhas & Chá & Tomar 3 vezes ao dia & Má digestão \\
\hline Bufa-do-cão & Raiz & $\begin{array}{l}\text { Faz-se um emplastro } \\
\text { com o pó }\end{array}$ & 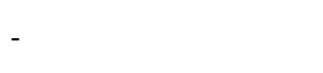 & Enfermidade \\
\hline Cajazeira-amarela & Casca do caule & Extrato aquoso & Tomar 3 vezes ao dia & Anemia \\
\hline Cajueiro-azedo e ameixa & Casca do caule & Chá & Tomar 3 vezes ao dia & Gastrite \\
\hline Capim-santo & Folhas & Chá & Tomar 3 vezes ao dia & Má digestão \\
\hline Catolé & Raiz & Chá & Tomar 3 vezes ao dia & Dor na coluna \\
\hline Chanana & Raiz & Chá & Tomar 3 vezes ao dia & Inflamação \\
\hline Ciúme & Caule & $\begin{array}{l}\text { Emplastro com o leite } \\
\text { do caule }\end{array}$ & $\begin{array}{l}\text { Manter o emplastro na } \\
\text { parte afetada até passar } \\
\text { os sintomas }\end{array}$ & Reumatismo \\
\hline Contra-erva & Raiz & Chá & Tomar 3 vezes ao dia & Gripe \\
\hline Cumaru & Casca do caule & Chá & Tomar 3 vezes ao dia & Tosse, bronquite e asma \\
\hline Courama & Folhas & Macerado & Coloca em cima & Tumor \\
\hline $\begin{array}{l}\text { Delegado com } \\
\text { malvarisco }\end{array}$ & Folhas & Chá & Tomar 3 vezes ao dia & Gripe \\
\hline Erva-cidreira & Folhas & Macerado & $\begin{array}{l}\text { Passar no local até passar } \\
\text { os sintomas }\end{array}$ & Enfermidade \\
\hline Fedegoso & Folhas & Chá & Até aliviar a tosse & Tosse \\
\hline Feijão-cuandu & Semente & $\begin{array}{l}\text { Faz-se um emplastro } \\
\text { com o pó }\end{array}$ & Até passar os sintomas & Inflamação \\
\hline Grão-de-galo & Caule & $\begin{array}{l}\text { Emplastro com o leite } \\
\text { do caule }\end{array}$ & $\begin{array}{l}\text { Manter o emplastro na } \\
\text { parte afetada até passar } \\
\text { os sintomas }\end{array}$ & Impingem \\
\hline Guabiraba & Folhas e casca & Chá & Continuamente & Câncer e inflamações \\
\hline Ipecacuanha & Raiz & Chá ou lambedor & Continuamente & Tosse \\
\hline Jurubeba-branca & Raiz & Chá & Tomar 3 vezes ao dia & Sangue e fígado \\
\hline Linhaça & Semente & Chá & Constantemente & Trombose e tumor \\
\hline Louco & Raiz & $\begin{array}{l}\text { Extrato hidroalcoól. } \\
\text { (com cachaça) }\end{array}$ & $\begin{array}{l}\text { Passar com algodão (no } \\
\text { dente afetado) }\end{array}$ & Dor de dente \\
\hline Malva & Folhas & Lambedor & Até aliviar a tosse & Tosse \\
\hline Manga itamaracá & Caroço & $\begin{array}{l}\text { Lambedor do caroço } \\
\text { pisado }\end{array}$ & $\begin{array}{l}\text { Beber até passar os } \\
\text { sintomas }\end{array}$ & Tuberculose \\
\hline Manjericão & Folhas & Chá & Até passar os sintomas & Gripe e banho \\
\hline Mamoeiro & Semente & Extrato aquoso & Tomar 3 vezes ao dia & Anti-helmíntico \\
\hline Maracujá & Folha & Chá & Tomar 3 vezes ao dia & Pressão alta \\
\hline Marmeleiro & Casca do caule & Chá & Tomar 3 vezes ao dia & Dor de estômago \\
\hline Mastruço ou mastruz & Folhas & Macerado & 3 vezes ao dia & Pneumonia e fratura \\
\hline Meracilina & Folhas & Chá & Tomar 3 vezes ao dia & Analgésico e antitérmico \\
\hline Pega-pinto e delegado & Folhas & $\begin{array}{l}\text { Extrato hidroalcoól. } \\
\text { (com cachaça) }\end{array}$ & Inalar 3 vezes ao dia & Gripe \\
\hline Quebra-pedra & Folha & Chá & Tomar 3 vezes ao dia & Cálculo renal \\
\hline Quiabo & Semente & Chá ou lambedor & Tomar 3 vezes ao dia & Fadiga pulmonar \\
\hline Rabo-de-tatu & Caule & $\begin{array}{l}\text { Extrato hidroalcoól. } \\
\text { (com cachaça) }\end{array}$ & Passar no local & Reumatismo \\
\hline Romã & Casca do fruto & Mastigação & Até passar os sintomas & Inflamação na garganta \\
\hline Velame & Folha & $\begin{array}{l}\text { Extrato hidroalcoól. } \\
\text { (com cachaça) }\end{array}$ & 3 vezes ao dia & Para o sangue \\
\hline Urucum & Semente & Lambedor & Até aliviar a tosse & Tosse \\
\hline
\end{tabular}

